\section{SAT0310 IDENTIFICATION OF FACTORS ASSOCIATED WITH MAGNETIC RESONANCE IMAGES CHANGES SUGGESTIVE OF AXIAL SPONDYLOARTHRITIS IN THE AXIAL SKELETON OF INDIVIDUALS $<45$ YEARS - EVALUATION OF DATA FROM A LARGE COMMUNITY STUDY}

Xenofon Baraliakos ${ }^{1}$, Adrian Richter ${ }^{2,3}$, Daniel Feldmann ${ }^{1}$, Anne Ott ${ }^{1}$, Robin Bülow ${ }^{4}$, Carsten Schmidt', Juergen Braun $1 .{ }^{1}$ Rheumazentrum Ruhrgebiet, Ruhr-University Bochum, Herne, Germany; ${ }^{2}$ Institute for Community Medicine, Greifswald, Germany; ${ }^{3}$ German Rheumatism Research Center, Berlin, Germany ${ }^{4}$ Department of Diagnostic Radiology and Neuroradiology, University Medicine, Greifswald, Germany

Background: Active (bone marrow edema, BME) and structural (fatty lesions, FL) lesions in the spinal and the sacroiliac joints (SIJ), as assessed by magnetic resonance imaging (MRI), have been described in patients with axial spondyloarthritis (axSpA) but may also occur in nonaxSpA patients and healthy individuals $(1,2)$.

Objectives: Identify factors associated with the occurrence of BME and FL in spinal- and SIJ-MRIs in the population-based Study of Health in Pomerania (SHIP).

Methods: All available spinal- (sagittal T1/T2 sequences) and SIJ- (semicoronal STIR sequences) MRIs were evaluated by two trained readers blinded to clinical data. BME (SIJ and spine) and FL (spine) suggestive of axSpA were recorded. Disagreements were resolved by consensus. BME was quantified using Berlin MRI scores. Clinical information was available for age (increase per decade), sex, smoking (ever vs. never), prevalence of spinal pain (NRS $\geq 4 / 10$ in last 3 months), CRP, HLA-B27 status and body mass index (BMI) categories (WHO definition of under(cat. 1), normal (cat. 2) or overweight (cat. 3)). Associations between clinical factors and MRI lesions were analyzed (a) by modeling of number and size of lesions using negative binomial count regression and (b) for presence/absence of lesions using univariate logistic regression.

Results: MRIs of 793 volunteers, 392 male (49.4\%), 45 CRP-positive (5.7\%), 67 HLA-B27+ (8.4\%), 497 smokers $(62.7 \%)$ were evaluated. BME lesions were found in 136 SIJ-MRIs (17.2\%) and in 218 (27.5\%) spinal MRIs, while FL were found in 645 spinal MRls (81.3\%).

A higher number and larger sized SIJ BME lesions were associated (exp $(\beta), 95 \% \mathrm{Cl})$ with HLA-B27 $(2.8,1.5-4.9)$, spinal pain $(1.7,1.2-2.6)$, and higher BMI (cat. 1 vs $3,1.7,1.0-3.0$ ). In the spine, higher number and larger sized BME were associated with age $(1.5,1.2-2.0)$, while $\mathrm{FL}$ were associated with higher BMI, (cat. 1 to cat. 3 (1.9, 1.5-2.2), age (1.6, 1.51.8) and male sex $(1.4,1.2-1.6)$.

Presence (odds ratio, $95 \% \mathrm{Cl}$ ) of spinal BME $(1.3,1.0-1.7)$ and/or $\mathrm{FL}$ (1.7, 1.3-2.3) was associated with age, while $\mathrm{FL}$ alone were associated with a higher BMI (cat. 1 to cat.3, 3.3, 1.8-6.1).

In total, 22 subjects (16.2\%) had large (>33\% of surface involvement) and 114 small $(\leq 33 \%$ of surface involvement) SIJ-BME lesions. Large SIJ-BME lesions were found in $4.5 \%$ HLA-B27-positive vs. $2.6 \%$ HLAB27-negative subjects $(p=0.40)$.

Out of 187 quadrants with BME lesions in SIJ, the majority $(n=66$, $35.3 \%$ ) was located in the upper sacral quadrant. Most SIJ lesions $(n=156,83.4 \%)$ were small. Out of 362 spinal segments with BME, the majority $n=152,42 \%)$ was located in the lower (T7/8-T11/12) thoracic spine. Most spinal lesions ( $n=329,90.9 \%)$ were small.

Conclusion: In this large population-based study, higher numbers of large SIJ-BME lesions were significantly associated with HLA-B27, back pain and BMI. In some contrast, spinal BME and FL were associated with older age, male sex and BMI but not HLA-B27. The association for HLA B27 was only shown on the quadrant but not on the patient level. These findings are in accordance with previously published hypotheses that question the specificity of sacroilitis and spondylitis in $\operatorname{axSpA~(3).~}$

\section{REFERENCES}

[1] Weber $U$ et al, Arthritis Rheumatol 2018

[2] De Winter J et al, Arthritis Rheumatol 2018

[3] Braun J et al, Rheumatology 2018

Disclosure of Interests: Xenofon Baraliakos Grant/research support from: AbbVie, Boehringer Ingelheim, Bristol-Myers Squibb, Celgene, Centocor, Chugai, Janssen, MSD, Novartis, Pfizer Inc, Roche and UCB, Grant/ research support from: AbbVie, Pfizer, Merck Sharp \& Dohme, UCB Pharma, Novartis, Consultant for: AbbVie, Bristol-Myers Squibb, Boehringer Ingelheim, Celgene, Chugai, Janssen Biologics, Novartis, Pfizer, UCB Pharma, Galapagos, Speakers bureau: AbbVie, Chugai, Janssen, Novartis, Pfizer, UCB Pharma, Adrian Richter: None declared, Daniel Feldmann: None declared, Anne Ott: None declared, Robin Bülow: None declared, Carsten Schmidt: None declared, Juergen Braun Shareholder of: AbbVie,
BMS, Celgene, Chugai, Merck, Novartis, Pfizer, UCB, Grant/research support from: AbbVie, BMS, Celgene, Chugai, Merck, Novartis, Pfizer, UCB, Grant/research support from: Abbott, Bristol Myers Squibb, Celgene, Celltrion, Chugai, Johnson \& Johnson, MSD, Novartis, Pfizer, Roche, UCB Pharma, Grant/research support from: AbbVie, BMS, Celgene, Chugai, Merck, Novartis, Pfizer, UCB, Grant/research support from: Abbvie (Abbott), Amgen, Baxter, Biogen, BMS, Boehringer, Celgene, Celltrion, Centocor, Chugai, Hexal, Janssen, Lilly, Medac, MSD (Schering-Plough), Mylan, Mundipharma, Novartis, Pfizer (Wyeth, Hospira), Roche, SanofiAventis and UCB, Consultant for: Abbvie (Abbott), Amgen, Baxter, Biogen, BMS, Boehringer, Celgene, Celltrion, Centocor, Chugai, Hexal, Janssen, Lilly, Medac, MSD (Schering-Plough), Mylan, Mundipharma, Novartis, Pfizer (Wyeth, Hospira), Roche, Sanofi-Aventis and UCB, Consultant for: AbbVie, BMS, Celgene, Chugai, Merck, Novartis, Pfizer, UCB, Consultant for: Abbott, Bristol Myers Squibb, Celgene, Celltrion, Chugai, Johnson \& Johnson, MSD, Novartis, Pfizer, Roche, UCB Pharma, Speakers bureau AbbVie, BMS, Celgene, Chugai, Merck, Novartis, Pfizer, UCB, Speakers bureau: Abbvie (Abbott), Amgen, Baxter, Biogen, BMS, Boehringer, Celgene, Celltrion, Centocor, Chugai, Hexal, Janssen, Lilly, Medac, MSD (Schering-Plough), Mylan, Mundipharma, Novartis, Pfizer (Wyeth, Hospira), Roche, Sanofi-Aventis and UCB, Speakers bureau: AbbVie, BMS, Celgene, Chugai, Merck, Novartis, Pfizer, UCB DOI: 10.1136/annrheumdis-2019-eular.4893

\section{SAT0311 DIAGNOSTIC UTILITY OF INDIVIDUAL INFLAMMATORY BACK PAIN PARAMETERS IN PATIENTS WITH AXIAL SPONDYLOARTHRITIS}

Manouk de Hooge ${ }^{1,2}$, Floris A. van Gaalen ${ }^{3}$, Marleen van de Sande ${ }^{4}$,

Karen Fagerli ${ }^{5}$, Lennart Jacobsson ${ }^{6}$, Dirk Elewaut ${ }^{1,2}$, Désirée van der Heijde ${ }^{3}$, Filip van den Bosch ${ }^{1,2}$. ${ }^{1}$ VIB Inflammation Research Center, Ghent, Belgium; ${ }^{2} U Z$ Gent, Ghent, Belgium; ${ }^{3}$ LUMC, Leiden, Netherlands; ${ }^{4}$ AMC, A'dam, Netherlands;

${ }^{5}$ Diakonhjemmet Hospital, Oslo, Norway, ${ }^{6}$ University of Göteborg, Göteborg, Sweden

Background: A recent study in German chronic back pain (CBP) patients (pts) with a suspicion of axial spondyloarthritis (axSpA) report on the performance of, among others, the ASAS inflammatory back pain (IBP) criteria and the individual IBP parameters. Pts were diagnosed by a rheumatologist blinded for all clinical features but IBP and by a rheumatologist with all diagnostic tests available as in daily practice (reference standard). Data show high sensitivity but low specificity of the ASAS IBP

\begin{tabular}{|c|c|c|c|c|c|c|}
\hline \multirow[b]{2}{*}{ 1a } & Be-Giant cohort & \multicolumn{2}{|c|}{ SPACE cohort } & \multicolumn{3}{|c|}{ SPACE cohort } \\
\hline & $\begin{array}{l}\text { axSpA } \\
\mathrm{N}=205\end{array}$ & \multicolumn{2}{|c|}{$\begin{array}{l}\text { axSpA } \\
\mathrm{N}=307\end{array}$} & \multicolumn{3}{|c|}{$\begin{array}{c}\text { No axSpA } \\
N=354\end{array}$} \\
\hline $\begin{array}{l}\text { IBP (ASAS definition), } \\
\text { n (\%) }\end{array}$ & $172(83.9)$ & \multicolumn{2}{|c|}{$228(74.3)$} & \multicolumn{3}{|c|}{$177(50.0)$} \\
\hline $\begin{array}{l}\text { Age at onset }<40 \\
\text { years }\end{array}$ & $197(96.1)$ & \multicolumn{2}{|c|}{$277(90.2)$} & \multicolumn{3}{|c|}{$312(88.1)$} \\
\hline Insidious onset & $185(90.2)$ & \multicolumn{2}{|c|}{$261(85.0)$} & \multicolumn{3}{|c|}{$298(84.2)$} \\
\hline $\begin{array}{l}\text { Improvement with } \\
\text { exercise }\end{array}$ & $181(88.3)$ & \multicolumn{2}{|c|}{$252(82.1)$} & \multicolumn{3}{|c|}{$228(64.4)$} \\
\hline $\begin{array}{l}\text { No improvement } \\
\text { with rest }\end{array}$ & $172(83.9)$ & \multicolumn{2}{|c|}{$264(86.0)$} & \multicolumn{3}{|c|}{$269(76.0)$} \\
\hline Pain at night & $162(79.0)$ & \multicolumn{2}{|c|}{$172(56.0)$} & \multicolumn{3}{|c|}{$131(37.0)$} \\
\hline \multicolumn{7}{|l|}{$\begin{array}{l}\text { No. of IBP parameters } \\
\text { present, } \mathbf{n}(\%)\end{array}$} \\
\hline 0 & 0 & \multicolumn{2}{|c|}{$1(0.3)$} & \multicolumn{3}{|c|}{0} \\
\hline 1 & $1(0.5)$ & \multicolumn{2}{|c|}{$5(1.6)$} & \multicolumn{3}{|c|}{$7(2.0)$} \\
\hline 2 & $7(3.4)$ & \multicolumn{2}{|c|}{$22(7.2)$} & & $6(15.8)$ & \\
\hline 3 & $25(12.2)$ & 51( & & & $14(32.2)$ & \\
\hline 4 & $53(25.9)$ & 1160 & & & $08(30.5)$ & \\
\hline 5 & $119(58.1)$ & 112( & & & $9(19.5)$ & \\
\hline th & $\begin{array}{r}\text { Sens } \\
\text { (confidenc }\end{array}$ & $\begin{array}{l}\text { ivity } \\
\text { interval) }\end{array}$ & $\begin{array}{r}\mathrm{S} \\
\text { (confic }\end{array}$ & & & \\
\hline to & Be-Giant cohort & SPACE cohort & & & $\begin{array}{l}\text { Be-Giant } \\
\text { cohort }\end{array}$ & $\begin{array}{l}\text { SPACE } \\
\text { cohort }\end{array}$ \\
\hline IBP (ASAS definition) & $\begin{array}{c}83.9 \%(78.0- \\
88.5)\end{array}$ & $\begin{array}{c}74.3 \%(68.9- \\
79.0)\end{array}$ & $50.0^{\circ}$ & & 1.7 & 1.5 \\
\hline $\begin{array}{l}\text { Age at onset }<40 \\
\text { years }\end{array}$ & $\begin{array}{l}96.1 \%(92.2- \\
98.2)\end{array}$ & $\begin{array}{c}90.2 \%(86.2- \\
93.2)\end{array}$ & $11.9^{\circ}$ & & 1.1 & 1.0 \\
\hline Insidious onset & $\begin{array}{c}90.2 \%(85.1- \\
93.8)\end{array}$ & $\begin{array}{c}85.0 \%(80.4- \\
88.7)\end{array}$ & $15.8^{\circ}$ & & 1.1 & 1.0 \\
\hline $\begin{array}{l}\text { Improvement with } \\
\text { exercise }\end{array}$ & $\begin{array}{c}88.3 \%(82.9- \\
92.2)\end{array}$ & $\begin{array}{c}82.1 \%(77.2- \\
86.1)\end{array}$ & $35.6^{\circ}$ & & 1.4 & 1.3 \\
\hline $\begin{array}{l}\text { No improvement } \\
\text { with rest }\end{array}$ & $\begin{array}{c}83.9 \%(78.0 \cdot \\
88.5)\end{array}$ & $\begin{array}{c}86.0 \%(81.5- \\
89.6)\end{array}$ & $24.0^{\circ}$ & & 1.1 & 1.1 \\
\hline Pain at night & $\begin{array}{c}79.0 \%(72.7- \\
84.3)\end{array}$ & $\begin{array}{c}56.0 \%(50.3- \\
61.6)\end{array}$ & $63.0^{\circ}$ & & 2.1 & 1.5 \\
\hline
\end{tabular}

Figure. Performance of individ ual parameters of the ASAS IBP criteria 